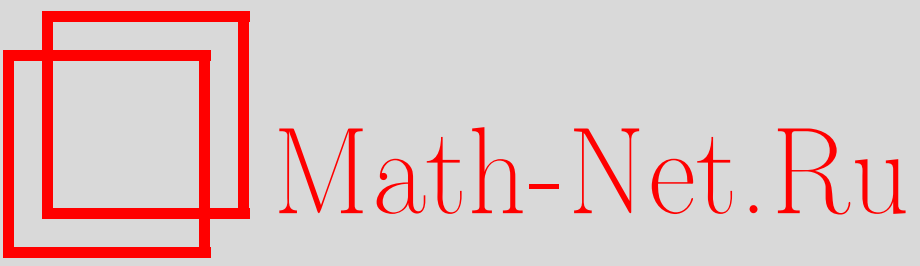

Г. В. Павлов, М. А. Кальмова, Эффект влияния полосы контакта упруговязкого основания на динамику диска, Вестн. Сам. гос. техн. ун-та. Сер. Физ.-мат. науки, 2009, выпуск 2(), 186-192

DOI: https://doi.org/10.14498/vsgtu693

Использование Общероссийского математического портала Math-Net.Ru подразумевает, что вы прочитали и согласны с пользовательским соглашением

http: //www. mathnet.ru/rus/agreement

Параметры загрузки:

IP : 54.174 .149 .18

26 апреля 2023 г., $11: 13: 54$ 
УДК 517.958:539.3(1)

\section{ЭФФЕКТ ВЛИЯНИЯ ПОЛОСЫ КОНТАКТА УПРУГОВЯЗКОГО ОСНОВАНИЯ НА ДИНАМИКУ ДИСКА}

\section{Г. В. Павлов, М. А. Калъмова}

Самарский государственный архитектурно-строительный университет, 443001, Самара, ул. Молодогвардейская, 194.

E-mail: yumishi@yandex.ru

Предлагается методика, учитывающая влияние горизонтальных деформаций реологического основания на движение жесткого кругового диска. Рассмотрен пример применения методики $к$ анализу движения диска по балке, наделённой деформационными и демпфирующими свойствами, отвечающими физической модели Кельвина.

Ключевые слова: неголономная связь, физическая модель Кельвина, время релаксаичи.

В связи с интенсивным применением синтетических материалов в машиностроении и строительстве возникает необходимость в усовершенствовании методов расчета неметаллических материалов, создании новых физических и математических моделей, наиболее полно описывающих характер работы упомянутых объектов. В настоящее время наблюдается повышенный интерес к применению реологических моделей при решении задач механики твёрдых деформированных тел, например [1,2]. Данная работа посвящена анализу движения однородного кругового жесткого диска по вязкоупругому основанию, отвечающему физической модели Кельвина. Рассмотрен эффект влияния местной деформации реологического основания на динамику диска. Если однородный диск движется плоскопараллельно и ускоренно, то следует учесть силы инерции и моменты сил инерции $[3,4]$.

В соответствии с принципом Даламбера имеют место следующие уравнения:

$$
\begin{gathered}
T-\Phi_{\mathrm{c}}-b \int_{0}^{t} \int_{\xi_{1}}^{\xi_{2}} \tau(\xi(t), t) d \xi d t=0, \quad Q-b \int_{0}^{t} \int_{\xi_{1}}^{\xi_{2}} p(\xi(t), t) d \xi d t=0, \\
T R-M_{z}^{\text {中 }}-M_{\mathrm{Tp}}-b \int_{0}^{t} \int_{\xi_{1}}^{\xi_{2}} \xi(t) p(\xi(t), t) d \xi d t=0 .
\end{gathered}
$$

Здесь $p$ и $\tau$ - удельные реакции основания на каток; $b$ - ширина катка, принимаемая равной единице; $\Phi_{\mathrm{c}}=m a_{\mathrm{c}}-$ сила инерции центра масс; $M_{z}^{\phi}=I_{z} \ddot{\varphi}-$ момент сил инерции, относительно оси, проходящей через центр масс; Tдвижущая или тормозящая сила; $M_{\text {тр }}$-момент трения качения; $\xi_{1}$ и $\xi_{2}-$ координаты граничных точек зоны контакта с катком; $R$-радиус диска;

Георгий Васильевич Павлов (к.ф.-м.н., доцент), доцент, каф. сопротивления материалов и строительной механики. Мария Александровна Кальмова, аспирант, каф. сопротивления материалов и строительной механики. 
$Q=m g-$ вес диска. В этих равенствах координаты $\xi_{1}$ и $\xi_{2}$ вследствие переменной скорости диска являются функциями времени. В процессе интегрирования допускаем упрощающее предположение, принимая время $t$ за постоянный параметр. Для разрешения интегральных соотношений (1) воспользуемся уравнениями $[4,5]$

$$
n \dot{p}+p=n E \dot{y}+\bar{E} y ; \quad y=y_{0}-\frac{1}{2 R} \xi^{2} ; \quad \dot{y}=\frac{V}{R} \xi ; \quad \dot{p}=-V \frac{d p}{d \xi},
$$

дополнив их соотношениями:

$$
\begin{gathered}
\dot{y}=\dot{y}_{0}-\frac{1}{R} \xi \dot{\xi}, \quad \dot{y}_{0}=\frac{1}{R} \xi_{2} \dot{\xi}_{2} \\
p(\xi, t)=E\left[y(t)-\frac{E-\bar{E}}{n E} \int_{0}^{t} y(\tau) \exp \left(\frac{t-\tau}{n}\right) d \tau\right],
\end{gathered}
$$

где $n$-время релаксации; $p$-вертикальная составляющая (напряжение) удельной силы реакции основания; $E$ и $\bar{E}-$ мгновенный и длительный модули упругости основания на растяжение; $y$ - прогиб основания, соответствующий координате $\xi ; y_{0}$ - максимальный прогиб основания под диском; $V$ - скорость мгновенного центра скоростей, точка означает дифференцирование по времени.

Равенство (4), представляющее собой интегральное уравнение с вырожденным ядром релаксации, является решением уравнения (2). Соотношение (4) с численными значениями параметров $E=1,5 \bar{E}$ и $n=50$ с соответствует физическим показателям стандартного наследственного тела. Такие параметры характерны для многих высоко эластичных синтетических материалов [1].

С учётом (2) и (4) определим нормальную силу $Q$ и момент нормальных усилий:

$$
\begin{gathered}
M=\frac{b E}{24 R}\left(2+\exp \left(-\frac{t}{n}\right)\right)\left(\xi_{1}^{2}-\xi_{2}^{2}\right)^{2}, \\
Q=\frac{b E}{24 R}\left(2+\exp \left(-\frac{t}{n}\right)\right)\left(\xi_{2}^{3}-3 \xi_{1} \xi_{2}^{2}+\xi_{1}^{2}\right) .
\end{gathered}
$$

Обращаясь к (2) и вспоминая, что $p\left(\xi_{1}\right)=0$, получим ещё одно уравнение, связывающее искомые величины $\xi_{1}$ и $\xi_{2}$ и их производные:

$$
\frac{\dot{\xi}}{3}\left(2+e^{-\frac{t}{50}}\right) \xi_{1}=\xi_{2} \dot{\xi}_{2}-\xi_{1} \dot{\xi_{1}}+\frac{\bar{E}}{2 n E}\left(\xi_{2}^{2}-\xi_{1}^{2}\right) .
$$

Здесь $\dot{\xi}$ - переменная скорость мгновенного центра скоростей диска. Для удобства анализа введём следующие безразмерные величины: $u=\xi_{1} / R, v=\xi_{2} / R$, $V=t \dot{\xi} / R, \dot{u}=t \dot{\xi}_{1} / R, \dot{v}=t \dot{\xi}_{2} / R$. Тогда соотношения (5), (6) примут вид

$$
\begin{gathered}
\frac{Q}{6 E R^{2}}=q=\frac{1}{18}\left(2+e^{-\beta}\right)\left(v^{3}-3 u v^{2}+u^{3}\right), \\
\frac{M}{6 E R^{3}}=m=\frac{1}{24}\left(2+e^{-\beta}\right)\left(u^{2}-v^{2}\right)^{2}, \\
\frac{1}{3}\left(2+e^{-\beta}\right) u V=v \dot{v}-u \dot{u} \frac{1}{2} k 3\left(u^{2}-v^{2}\right),
\end{gathered}
$$


где $q, m, \beta=t / 30$ - безразмерные величины.

Уравнения (7)-(9) полностью решают задачу об определении реакций диска на вязкоупругом основании.

В качестве примера применения полученных уравнений рассмотрим задачу о движении однородного диска массой $m$ и радиусом $R$ по безмассовой бесконечно длинной балке жесткостью $c_{1}$ на вязкоупругом основании, моделируемом системой параллельных вертикальных пружин, обладающих реологическими свойствами. Это позволяет представить физическую модель как дискретную систему, движение которой описывается системой обыкновенных дифференциальных уравнений.

Ориентацию диска в системе $O \xi \eta \zeta$ определим при помощи четырех координат: $\alpha, \varphi, \gamma$ и $\rho$. Угол $\gamma$ характеризует степень наклона касательной к упругой линии балки. Координаты $\alpha, \varphi$ и $\gamma$ - генеральные, координата $\rho-$ реологическая, обусловленная деформацией сжатия винклеровского основания. Она является избыточной и зависимой, так как может быть известным образом найдена в функции угла $\gamma$. Но для удобства представления уравнений движения именно в дифференциальной форме принимаем координату $\rho$ за обобщённую. Введены четыре системы координат - неподвижная $O \xi \eta \zeta$ и подвижные: $D \xi^{*} \eta^{*} \zeta^{*}$, оси которой параллельны осям $O \xi \eta \zeta$, система $D \tau n b$, которая при движении вершины $D$ (точки касания диска с балкой) вращается с угловой скоростью $\dot{\gamma}$ вокруг оси $b$, параллельной оси $\xi$, и полуподвижная система $D x y z$, ось $x$ которой ортогональна плоскости диска. Положение плоскости диска по отношении к осям $D \tau n b$ определим углом $\alpha$, образуемым вращением диска вокруг оси $\tau$.

Так как при движении диска система осей $\tau, n$ вращается против часовой стрелки, то имеет место равенство $|\dot{\gamma}|=\dot{\gamma}$.

Проекции абсолютной угловой скорости диска на подвижные и неподвижные оси определяются равенствами

$$
\begin{gathered}
\omega_{x}=-\dot{\varphi}+\dot{\gamma} \cos \alpha, \quad \omega_{y}=\dot{\alpha}, \quad \omega_{z}=\dot{\gamma} \sin \alpha, \quad \omega_{\xi}=\dot{\gamma}-\dot{\varphi} \cos \alpha \\
\omega_{\eta}=-\dot{\varphi} \sin \alpha \sin \gamma+\dot{\alpha} \cos \gamma, \quad \omega_{\zeta}=\dot{\varphi} \sin \alpha \cos \gamma-\dot{\alpha} \sin \gamma
\end{gathered}
$$

Условие качения диска по балке без проскальзывания в точке касания $D$ выражено векторным равенством

$$
\boldsymbol{V}_{D}=\frac{d}{d t}\left(r \varphi \boldsymbol{\eta}_{0}-\rho \boldsymbol{\zeta}_{0}+r \boldsymbol{z}_{0}\right)+\boldsymbol{\omega} \times \boldsymbol{\chi}=\boldsymbol{V}_{\rho},
$$

где $\boldsymbol{\omega}=\omega_{\xi} \boldsymbol{\xi}_{0}+\omega_{\eta} \boldsymbol{\eta}_{0}+\omega_{\zeta} \boldsymbol{\zeta}_{0}, \boldsymbol{\chi}=-r\left(\sin \alpha \boldsymbol{\xi}_{0}+\cos \alpha \sin \gamma \boldsymbol{\eta}_{0}+\cos \alpha \cos \gamma \boldsymbol{\zeta}_{0}\right), \boldsymbol{\xi}_{0}$, $\boldsymbol{\eta}_{0}, \boldsymbol{\zeta}_{0}, \boldsymbol{z}_{0}$ - единичные векторы соответствующих осей, $\boldsymbol{V}_{\rho}$-вертикальная составляющая скорости балки в точке $D$.

Проектируя (11) на неподвижные оси $O \xi \eta \zeta$, находим уравнения связей:

$$
\begin{gathered}
\Phi_{1}(\alpha, \gamma, \dot{\varphi}, \dot{\gamma})=\dot{\varphi}-\cos \gamma(\dot{\gamma} \cos \alpha+\dot{\varphi})=0, \\
\Phi_{2}(\alpha, \gamma, \dot{\varphi}, \dot{\gamma}, \dot{\rho})=-\dot{\rho}+r \sin \gamma(\dot{\varphi} \cos 2 \alpha+\dot{\gamma} \cos \alpha)=0, \\
\Phi_{3}(\alpha, \gamma, \dot{\varphi})=\dot{\varphi} \sin 2 \alpha \sin 2 \gamma=0 .
\end{gathered}
$$

Поскольку выполняются неравенства $\frac{\partial^{2} \Phi_{i}}{\partial \dot{\varphi} \partial \gamma} \neq \frac{\partial^{2} \Phi_{i}}{\partial \dot{\gamma} \partial \varphi}(i \equiv 1,2)$, то первые два уравнения (12) представляют собой неинтегрируемые соотношения и являются уравнениями неголономных связей. 
Последнее уравнение связи (12) накладывает ограничение на характер движения диска, оставляя ему свободу передвижения в вертикальной плоскости, что совпадает с классическим представлением о движении диска по горизонтальной прямой. Поэтому в дальнейшем угловая координата $\alpha$ исключается из рассмотрения.

Координаты точки касания диска и проекции силы $\boldsymbol{P}$ на оси системы $O \xi \eta \zeta$ удовлетворяют следующим равенствам: $\xi_{D}=0, \eta_{D}=r \varphi, \zeta_{D}=-\rho$, $P_{\xi}=0, P_{\eta}=P \sin \gamma, P_{\zeta}=P \cos \gamma$.

Кинетическая и потенциальная энергии диска имеют следующий вид:

$$
\begin{gathered}
T=\frac{m V_{D}^{2}}{2}+m\left|\begin{array}{ccc}
\omega_{x} & \omega_{y} & \omega_{z} \\
x_{D} & y_{D} & z_{D} \\
V_{D x} & V_{D y} & V_{D z}
\end{array}\right|+\frac{1}{2}\left(J_{x} \omega_{x}^{2}+J_{y} \omega_{y}^{2}+J_{z} \omega_{z}^{2}\right), \\
\Pi=c_{1} \int_{-\rho}^{0} y d y \int_{-\gamma}^{0} \cos ^{2} z d z-m g[r(1-\cos \gamma)+\rho],
\end{gathered}
$$

где положено $J_{x}=3 m r^{2} / 2, J_{y}=5 m r^{2} / 4, J_{z}=m r^{2} / 4 ; x_{D}=y_{D}=0, z_{D}=r$, $\boldsymbol{\omega}=(-\dot{\varphi}+\dot{\gamma}, 0,0), V_{D x}, V_{D y}, V_{D z}$ - проекции скорости точки $D$ на оси полуподвижной системы координат.

В силу консервативности системы структура обобщённых сил полностью определяется выражением потенциальной энергии:

$$
Q_{\rho}=-\frac{\partial \Pi}{\partial \rho}, \quad Q_{\gamma}=-\frac{\partial \Pi}{\partial \gamma}, \quad Q_{\varphi}=0
$$

Силовыми факторами, учтенными в задаче, в соответствии с рис. 1 являются реакция $P$ реологического основания, сила реакции упругой балки $R$ и сила тяжести диска $m g$.

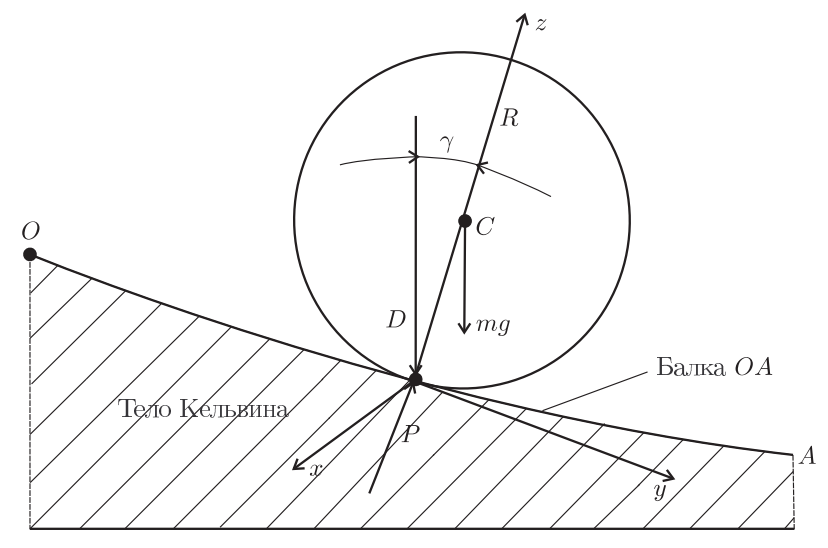

Рис. 1. Схема качения диска

Сумма $\sum_{k=1}^{K} b_{k j} P_{k}=\sum_{i=1}^{3 N} P_{i} \frac{\partial x_{i}}{\partial q_{j}}(j \equiv 1,2, \ldots, m)$ определена как $j$-тая обобщённая сила, соответствующая $j$-той обобщённой генеральной координате 
для $k$-той реологической силы реакции по формулам

$$
\begin{gathered}
\sum_{i=1}^{3} b_{i \varphi} P_{i}=P_{\xi} \frac{\partial \xi_{D}}{\partial \varphi}+P_{\eta} \frac{\partial \eta_{D}}{\partial \varphi}+P_{\zeta} \frac{\partial \zeta_{D}}{\partial \varphi}=\operatorname{Pr} \sin \gamma \\
\sum_{i=1}^{3} b_{i \gamma} P_{i}=P_{\xi} \frac{\partial \xi_{D}}{\partial \gamma}+P_{\eta} \frac{\partial \eta_{D}}{\partial \gamma}+P_{\zeta} \frac{\partial \zeta_{D}}{\partial \gamma}=0 .
\end{gathered}
$$

Уравнения движения диска, записанные в форме Рауса с множителями связей, принимают следующий вид:

$$
\begin{gathered}
\frac{d}{d t} \frac{\partial T}{\partial \dot{\varphi}}-\frac{\partial T}{\partial \varphi}-Q_{\varphi}+P r \sin \gamma+\lambda_{1} r(\cos \gamma-1)-\lambda_{2} r \sin \gamma=0 \\
\frac{d}{d t} \frac{\partial T}{\partial \dot{\gamma}}-\frac{\partial T}{\partial \gamma}-Q_{\gamma}+\lambda_{1} r \cos \gamma-\lambda_{2} r \sin \gamma=0 \\
\frac{d}{d t} \frac{\partial T}{\partial \dot{\rho}}-\frac{\partial T}{\partial \rho}-Q_{\rho}+P \cos \gamma+\lambda_{2}=0
\end{gathered}
$$

В соответствии с определением суммы $\sum_{k=1}^{K} b_{k j} P_{k}$ при нахождении обобщённой силы $Q_{\rho}$, в неё не должна входить возможная работа, производимая реологической силой $P$.

Решая совместно первое и третье уравнения системы (13), исключаем реакцию основания $P$ из первого уравнения. Решая совместно третье уравнение системы $(11)$ с $(2)$, исключаем реакцию $P$ из уравнения $(2)$. Тогда система (13) примет вид

$$
\begin{gathered}
\frac{d}{d t} \frac{\partial T}{\partial \dot{\varphi}}-\frac{\partial T}{\partial \varphi}-Q_{\varphi}-r \operatorname{tg} \gamma\left[\frac{d}{d t} \frac{\partial T}{\partial \dot{\rho}}-\frac{\partial T}{\partial \rho}-Q_{\rho}+\lambda_{2}\right]+ \\
\quad+r \lambda_{1}(\cos \gamma-1)-r \lambda_{2} \sin \gamma=0 \\
\frac{d}{d t} \frac{\partial T}{\partial \dot{\gamma}}-\frac{\partial T}{\partial \gamma}-Q_{\gamma}+r \lambda_{1} \cos \gamma-r \lambda_{2} \sin \gamma=0 \\
\left(1+n \frac{d}{d t}\right)\left[\frac{1}{\cos \gamma}\left(\frac{d}{d t} \frac{\partial T}{\partial \dot{\rho}}-\frac{\partial T}{\partial \rho}-Q_{\rho}+\lambda_{2}\right)\right]+n c \dot{\rho}+\tilde{c} \rho=0
\end{gathered}
$$

Разрешая первое и второе уравнения системы (14) относительно множителей связей, определим $\lambda_{2}$. Окончательно решение задачи в дифференциальной форме сводится к интегрированию системы уравнений, состоящей из уравнения третьего порядка и двух уравнений связей:

$$
\begin{gathered}
\left(1+n \frac{d}{d t}\right)\left[\frac{1}{\cos \gamma}\left(\frac{d}{d t} \frac{\partial T}{\partial \dot{\rho}}-\frac{\partial T}{\partial \rho}-Q_{\rho}+f\right)\right]+n c \dot{\rho}+\tilde{c} \rho=0 \\
\dot{\varphi}(1-\cos \gamma)-\dot{\gamma} \cos \gamma=0, \quad-\dot{\rho}+r \sin \gamma(\dot{\varphi}+\dot{\gamma})=0 .
\end{gathered}
$$


Здесь $f$ - функция, зависящая от координат $\varphi, \gamma, \rho$ и их производных.

Путём численного решения системы уравнений (15) совместно с уравнениями (6), (8) был проведён анализ режима движения диска при следующих эксплуатационных данных и начальных условиях: $\omega=20 \mathrm{c}^{-1}, n=50 \mathrm{c}, \bar{E}=$ $=0,7 E, E=70 \mathrm{H} / \mathrm{M}, c_{1}=50 \mathrm{H} / \mathrm{M}, r=0,2 \mathrm{M}, t_{k}=1500 \mathrm{c}, g=10 \mathrm{~m} / \mathrm{c}^{2}$, $\gamma(0)=0,01$ рад, $\rho(0)=0, \varphi(0)=0, \dot{\varphi}(0)=20 c^{-1}, \ddot{\varphi}(0)=0,1$. Здесь $t_{k}$ - время движения диска.

На рис. 2 представлена картина протекания реологического процесса. Из анализа графических зависимостей следует, что происходит процесс торможения диска, и его энергия переходит в потенциальную энергию деформированной балки. После остановки начинается движение балки с диском вверх и качение диска в обратном направлении до момента исчезновения деформации балки и т. д. Учёт момента трения качения вносит наибольший вклад в упругую составляющую основания, увеличивая его жесткость, что приводит к возникновению высокочастотных колебаний. При этом наблюдается совпадение частоты вертикальных колебаний диска с частотами граничных точек контактной зоны, которая в процессе всего движения диска в точку не стягивается, что указывает на отсутствие фазы отрыва диска. Диск будет постоянно менять направление движения по балке, совершая затухающие маятниковые колебания. Наблюдается ожидаемый результат, при котором изменение быстроосцилирующей вертикальной координаты точки касания диска приводит к горизонтальным деформациям основания, определяемым координатами $\xi_{1}$ и $\xi_{2}$ (рис. 2, а, б). Вместе с тем сокращается время переходного режима от начала движения диска до его остановки.

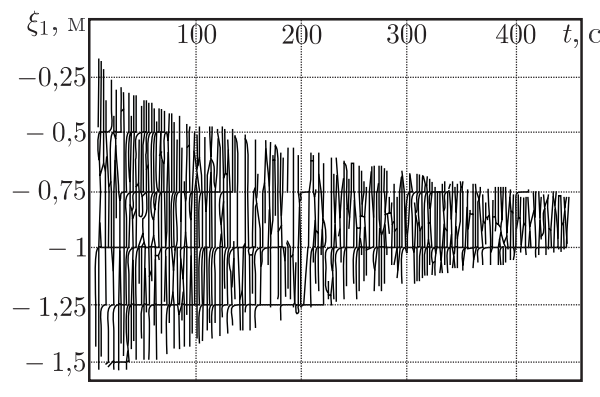

a

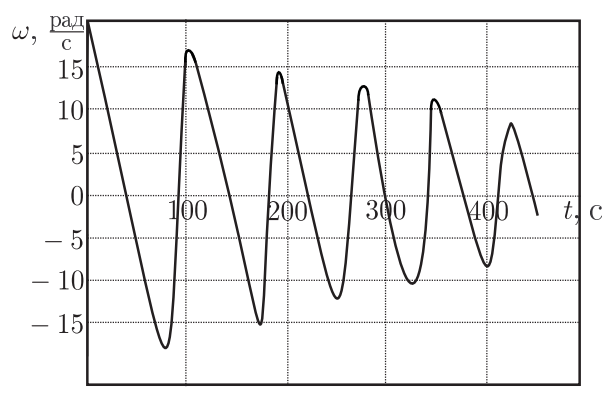

$B$

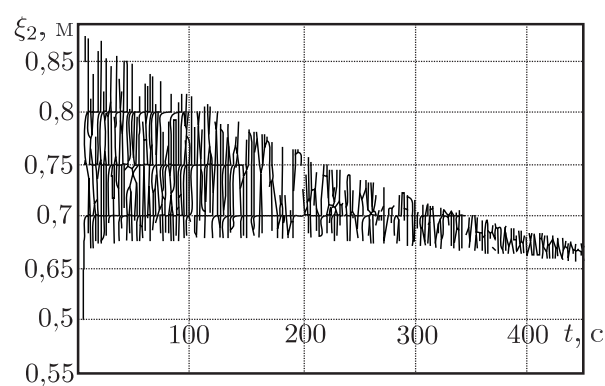

б

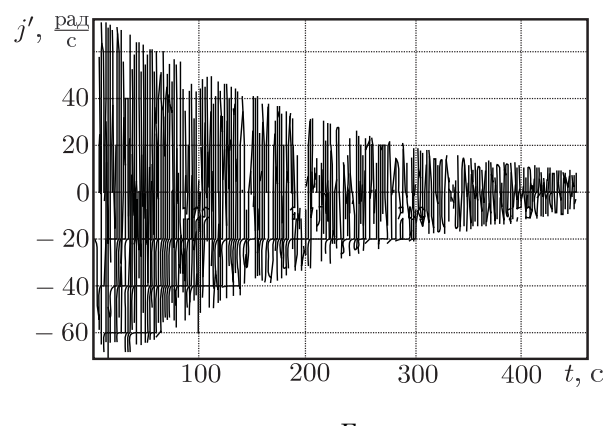

$\Gamma$

Рис. 2. Графики изменения: а-начала контактной зоны; б-конца контактной зоны; вугловой скорости без учета момента трения качения; $\Gamma$ - угловой скорости с учетом момента трения качения 


\section{БИБЛИОГРАФИЧЕСКИЙ СПИСОК}

1. Хедрuх К. С. Аналитичка динамика (механика) дискретних наследних система (на сербск. яз.). - Универзитет у Нишу, 2001.

2. Ульчинкова В. Э., Кухаръ В. Д. Реологические модели обобщённых сплошных сред. Тула: ТулГУ, 2005. - 140 с.

3. Иилинский А. Ю. Механика, идеи, задачи, приложения. - М.: Наука, 1985. - 623 с.

4. Иилинский А.Ю. Трение качения// Прикладная математика и механика, 1938. T. 2, № 2. - C. 245-260.

5. Ржанищын A. Р. Некоторые вопросы механики систем, деформирующихся во времени. - М.: Гостехиздат, 1949. - 248 с.

Поступила в редакцию 26/V/2009;

в окончательном варианте - 10/XI/2009.

MSC: 37N15, 74H40, 74F99

\section{CONTACT LINE INFLUENCE EFFECT OF VISCOELASTIC SUB-BASE ON THE DISK DYNAMICS}

\section{G. V. Pavlov, M. A. Kal'mova}

Samara State University of Architecture and Civil Engineering, 194, Molodogvardeyskaya str., Samara, 443001.

E-mail: yumishi@yandex.ru

The article deals with the method that considers rolling friction moment of a rheological sub-base effect on the movement of firm circular disk. The authors give an example of this method application to analysis of the movement of the disk on the hummer brace, that has got deformation and damping properties responding to Kelvin model.

Key words: nonholonomic constraint, rheological Kelvin model, relaxation time.

Original article submitted $26 / \mathrm{V} / 2009$; revision submitted 10/XI/2009.

Georgy V. Pavlov (Ph. D. (Phys. \& Math.)), Associate Professor, Dept. of Resistance of Materials \& Construction Mechanics. Mariya A. Kal'mova, Postgraduate Student, Dept. of Resistance of Materials \& Construction Mechanics. 